Article

\title{
Reduction of Homogeneous Pseudo-Kähler Structures by One-Dimensional Fibers
}

\author{
José Luis Carmona Jiménez * and Marco Castrillón López \\ Departamento de Álgebra, Geometría y Topología, Facultad de Matemáticas, Universidad Complutense \\ de Madrid, 28040 Madrid, Spain; mcastri@mat.ucm.es \\ * Correspondence: jcarmo03@ucm.es
}

Received: 18 June 2020; Accepted: 30 July 2020; Published: 1 August 2020

\begin{abstract}
We study the reduction procedure applied to pseudo-Kähler manifolds by a one dimensional Lie group acting by isometries and preserving the complex tensor. We endow the quotient manifold with an almost contact metric structure. We use this fact to connect pseudo-Kähler homogeneous structures with almost contact metric homogeneous structures. This relation will have consequences in the class of the almost contact manifold. Indeed, if we choose a pseudo-Kähler homogeneous structure of linear type, then the reduced, almost contact homogeneous structure is of linear type and the reduced manifold is of type $\mathcal{C}_{5} \oplus \mathcal{C}_{6} \oplus \mathcal{C}_{12}$ of Chinea-González classification.
\end{abstract}

Keywords: Ambrose-Singer connections; almost contact metric manifolds; homogeneous manifolds; homogeneous structures; pseudo-Kähler manifolds; pseudo-Riemannian metrics

\section{Introduction}

First, Ambrose and Singer [1] gave a tensorial approach to study homogeneous Riemannian manifolds by the so-called homogeneous structure $S$. Later, Kiričenko [2] extended this approach to homogeneous Riemannian manifolds with additional geometric structures. Furthermore, references [3-5] decomposed the space of tensors $S$ in cases of the additional geometry being purely Riemannian, Kähler or almost contact metric, respectively.

Nowadays, the application of homogeneous structures is a main tool in the investigation of homogeneous manifolds; see [3,5], among others. Moreover, homogeneous manifolds are a central object for many mathematical models of physical theories (for example, linear degenerate homogeneous structures are related to homogeneous plane waves; cf. [6]). This is specially relevant when the space is also equipped with additional geometry, such as contact or Kähler. Nevertheless, the scare knowledge about the relationships between homogeneous structures when there is a map between homogeneous manifolds is remarkable. An example of this is the reduction procedure of homogeneous structures, which was first introduced in [7], wherein, in particular, the authors reduced pseudo-Riemannian almost contact homogeneous structures to pseudo-Riemannian, almost-Hermitian homogeneous structures.

In this paper, we examine the reduction procedure of a pseudo-Kähler homogeneous manifold to almost contact metric homogeneous manifolds by one dimensional fibers. We show that the almost contact metric manifold is of type $\mathcal{C}_{5} \oplus \mathcal{C}_{6} \oplus \mathcal{C}_{7} \oplus \mathcal{C}_{8} \oplus \mathcal{C}_{9} \oplus \mathcal{C}_{10} \oplus \mathcal{C}_{12}$ of Chinea-González classification (cf. [8]).

Some of the most important cases of homogeneous structures are those ones of linear type, in which the dimension of the class grows linearly with the dimension of the manifold; see ([5] Chapter 5). Linear classes always provide, in the different geometries with which they have been studied, interesting results, from the characterization of negative constant curvature (cf. [3] Theorem 5.2) in Riemannian manifolds, to other surprising facts in other geometries (see [9] for a 
survey). In our work, we show that if the Kähler manifold has a homogeneous structure of linear type, then the reduced homogeneous structure is of linear type. Furthermore, as the homogeneous structures of almost contact metric manifolds are related with the covariant derivative of the fundamental 2-form associated to it, we prove that the reduced manifold by a homogeneous linear structure is of type $\mathcal{C}_{5} \oplus \mathcal{C}_{6} \oplus \mathcal{C}_{12}$ of Chinea-González classification (cf. [8]). Besides that, if the one-dimensional Lie group is proportional to the sum of the two vectors that defines each projection to the subspaces $\mathcal{K}_{2} \oplus \mathcal{K}_{4}$ of the linear homogeneous structure, then the manifold is Sasakian. Moreover, the reduced manifold is cosymplectic if the sum is zero.

\section{Preliminaries}

\subsection{Homogeneous Structures}

For a general non-metric framework of homogeneous structures, see [10]. Here, we will focus on the well known notion of pseudo-Riemannian Homogeneous structures that we summarize as follows (see [5] and the references therein for a detailed description). Let $(M, g)$ be a connected pseudo-Riemannian manifold and let $\nabla$ be the Levi-Civita connection. A $(1,2)$-tensor field $S$ in $M$ is said to be a pseudo-Riemannian homogeneous structure if and only if it satisfies

$$
\widetilde{\nabla} R=0, \quad \widetilde{\nabla} g=0, \quad \widetilde{\nabla} S=0,
$$

where $\widetilde{\nabla}=\nabla-S$ and $R$ is the curvature form of $\nabla$. A manifold $(M, g)$ is reductive and locally homogeneous if and only if it is endowed with a pseudo-Riemannian homogeneous structure (cf. [11]). It is sometimes more convenient to work with $(0,3)$-tensors instead of $(1,2)$-tensors with the relation

$$
\left(S_{p}\right)_{X Y Z}=g\left(\left(S_{p}\right)_{X} Y, Z\right), \quad X, Y, Z \in V
$$

For the sake of simplicity, both tensor fields will be denoted the same. If we fix a point $p \in M$ and we consider $V=T_{p} M$, the condition $\widetilde{\nabla} g=0$ implies that $S_{p}$ belongs to the space

$$
\mathcal{S}(V)=\left\{S \in \otimes^{3} V: S_{X Y Z}=-S_{X Z Y}\right\}
$$

The decomposition of this space into irreducible components under the action of the orthogonal group provides a set of classes such that $S_{p}$ belongs to the same class for every choice of the point $p$. If the manifold $(M, g)$ is also equipped with an additional geometric structure defined by a tensor $K$, then the condition $\widetilde{\nabla} K=0$ must be included in (1) to characterize the reductive local homogeneity of the manifold under (local) transformations preserving both $g$ and $K$. We apply this situation to two instances:

- Let $(M, g, J)$ be an almost pseudo-Hermitian manifold, that is, a pseudo-Riemannian manifold equipped with a $(1,1)$-tensor $J$ that is a point-wise isometry. A pseudo-Hermitian homogeneous structure $S$ is a $(1,2)$-tensor satisfying $(1)$ and $\widetilde{\nabla} J=0$. If in addition $(M, g, J)$ is Kähler (that is, $\nabla J=0)$, and we fix a point $p \in M, V=T_{p} M$, the linear space of tensors to be considered is

$$
\mathcal{K}(V)=\left\{S \in \otimes^{3} V: S_{X Y Z}=-S_{X Z Y}, \quad S_{X J Y J Z}=S_{X Y Z}\right\},
$$

obtained by implementing the condition $\widetilde{\nabla} J=-S J=0$ to $\mathcal{S}(V)$. It is proven in [4,9] that the space $\mathcal{K}(V)$ of pseudo-Kähler homogeneous structures decomposes in four mutually orthogonal and irreducible $\mathrm{U}(p, q)$-submodules

$$
\mathcal{K}(V)=\mathcal{K}_{1}(V) \oplus \mathcal{K}_{2}(V) \oplus \mathcal{K}_{3}(V) \oplus \mathcal{K}_{4}(V)
$$

Their expressions can be found in the Appendix A. 
- Let $(M, g, \phi, \xi, \eta)$ be an almost contact metric manifold, that is (for example, see [12]), a pseudo-Riemannian manifold $(M, g)$ equipped with a $(1,1)$-tensor $\phi$ and a vector field $\xi$ (the 1-form $\eta$ being its dual with respect to $g$ ) such that

$$
\phi^{2}=-\mathrm{id}+\eta \otimes \xi, \quad g(\phi(X), \phi(Y))=g(X, Y)-\varepsilon \eta(X) \eta(Y)
$$

where $\varepsilon=g(\xi, \xi)$. Then, $S$ is a almost contact metric homogeneous structure if and only if (1) is satisfied together with $\widetilde{\nabla} \phi=0$. This implies that also $\widetilde{\nabla} \xi=0$ and $\widetilde{\nabla} \eta=0$. The equation $\widetilde{\nabla} \phi=0$ is equivalent to $\nabla \phi=[S, \phi]$, but this condition cannot be easily implemented into the definition of the pointwise space of tensors $\mathcal{S}(V), V=T_{p} M$, since the Levi-Civita connection depends on the metric and the first derivatives of the metric. However, we can still split this space of linear tensors under the group $\mathrm{U}(r, s) \times 1$. Recall that this group characterizes the canonical almost metric structure of $\mathbb{R}^{2 n+1}$ defined by $\xi_{0}=e_{2 n+1}$ and

$$
\phi_{0}=\left(\begin{array}{cc}
J_{0} & 0 \\
0 & 0
\end{array}\right),
$$

$J_{0}$ being the standard complex structure of $\mathbb{R}^{2 n}$. That is, $\mathrm{U}(r, s) \times 1$ is the subgroup of $O(2 r+1,2 s)$ or $O(2 r, 2 s+1)$ (depending on the value of $\varepsilon$ ) stabilizing both $\xi_{0}$ and $\phi_{0}$. Then, $\mathcal{S}(V)$ decomposes into two mutually orthogonal $\mathrm{U}(r, s) \times 1$ submodules,

$$
\mathcal{S}(V)=\mathcal{S}_{+}(V) \oplus \mathcal{S}_{-}(V) .
$$

with

$$
\begin{aligned}
& \mathcal{S}_{+}(V)=\left\{S \in \mathcal{S}(V): S_{X \phi Y \phi Z}=S_{X Y Z}\right\} \\
& \mathcal{S}_{-}(V)=\left\{S \in \mathcal{S}(V): S_{X \phi Y \phi Z}+S_{X Y Z}=\eta(Y) S_{X \xi Z}+\eta(Z) S_{X Y \xi}\right\}
\end{aligned}
$$

Additionally, these two submodules decompose in mutually orthogonal and irreducible $\mathrm{U}(p, q) \times 1$-submodules

$$
\begin{aligned}
& \mathcal{S}_{+}(V)=\mathcal{C S}_{1}(V) \oplus \cdots \oplus \mathcal{C S}_{6}(V) \\
& \mathcal{S}_{-}(V)=\mathcal{C}_{1}(V) \oplus \cdots \oplus \mathcal{C}_{12}(V)
\end{aligned}
$$

where the first classification is given in ([5] Prop. 4.2.10) and the second in [8]. See the Appendix A for their expressions. Cosymplectic manifolds are an important subcase of almost contact metric manifolds. They are characterized by the additional condition $\nabla \phi=0$. Hence, a homogeneous structure $S$ belongs to $\mathcal{S}_{+}(V)$ if and only if the manifold is cosymplectic.

\subsection{Reduction of a Homogeneous Structure}

Let $\pi: \bar{M} \longrightarrow M$ be a (left) $G$-principal bundle, where $\bar{M}$ is a pseudo-Riemannian manifold with metric $\bar{g}$, and the fibers are non-degenerate with respect to $\bar{g}$. Suppose that $G$ acts on $\bar{M}$ by isometries.

Given $\bar{p}$, we consider $V_{\bar{p}} \bar{M}$ the vertical subspace at $\bar{p}$ and $H_{\bar{p}} \bar{M}$ its orthogonal complement with respect to $\bar{g}$. As $G$ acts by isometries, the decomposition

$$
T_{\bar{p}} \bar{M}=V_{\bar{p}} \bar{M} \oplus H_{\bar{p}} \bar{M}
$$

is a principal G-connection. This connection $\omega$ is sometimes called a mechanical connection for its relevant role in some problems in geometric mechanics (see [13]). Furthermore, there is a unique 
pseudo-Riemannian metric $g$ in $M$ such that the restriction $\pi_{*}: H_{\bar{p}} \bar{M} \longrightarrow T_{\pi(\bar{p})} M$ is an isometry for every $\bar{p} \in \bar{M}$. Obviously, the metric $g$ satisfies

$$
g(X, Y) \circ \pi=\bar{g}\left(X^{H}, Y^{H}\right) \quad \text { for all } X, Y \in \mathfrak{X}(M),
$$

where $X^{H}$ and $Y^{H}$ denote the horizontal lift of $X$ and $Y$ with respect to the mechanical connection.

Let $\bar{\nabla}$ be the Levi-Civita connection and $\bar{S}=\bar{\nabla}-\widetilde{\bar{\nabla}}$ be a pseudo-Riemannian homogeneous structure on $\bar{M}$, invariant under the action of the structure group $G$. Assume that there is 1 -form $\beta$ taking values in End $(\mathfrak{h})$ such that

$$
\widetilde{\nabla} \omega=\beta \cdot \omega .
$$

Then, by ([7] Theorem 3.7), the reduced tensor field $S$ on $M$ defined by

$$
S_{X} Y=\pi_{*}\left(\bar{S}_{X^{H}} Y^{H}\right), \quad X, Y \in \mathfrak{X}(M),
$$

is a pseudo-Riemannian homogeneous structure of $(M, g)$.

\section{Fibrations of Pseudo-Hermitian over Almost Contact Metric Manifolds}

Let $\theta$ be a nowhere-vanishing vector field in $\bar{M}$. Around any point $\bar{p} \in \bar{M}$ there exists a coordinate system $\left(x^{1}, \ldots, x^{m}\right), m=\operatorname{dim} \bar{M}$, in a neighborhood diffeomorphic to $[0,1]^{m}$ such that any integral curve of $\theta$ is given by $x^{1}=$ const $, \ldots, x^{m-1}=$ const. The vector field is said to be regular if the domains can be always chosen such that any orbit of $\theta$ intersects them at most once. For regular vector fields, the orbit space $M$ is a smooth manifold and the projection $\pi: \bar{M} \longrightarrow M$ a submersion (cf. [14]). Furthermore, a regular vector field is said to be strictly regular if all the orbits are diffeomorphic. If $\theta$ is a complete, strictly regular vector field, the one-parametric group $G$ generated by $\theta\left(G=\mathbb{R}\right.$ or $\left.S^{1}\right)$ acts freely on $\bar{M}$, and $\pi: \bar{M} \longrightarrow M$ is a G-principal bundle. If we further assume that $\bar{M}$ is equipped with a pseudo-Riemannian metric $\bar{g}$ such that $\bar{g}(\theta, \theta)= \pm 1$ (that is, $\theta$ is non-degenerate so that we can normalize it) and $\bar{g}$ is invariant by the group $G$, then the 1 -form

$$
\omega(v)=\varepsilon \bar{g}(\theta, v), \quad v \in T \bar{M},
$$

where $\varepsilon=\operatorname{sign}(\bar{g}(\theta, \theta))$ is a $G$-principal connection form in $\pi: \bar{M} \longrightarrow M$, a mechanical connection, as we mentioned above.

Theorem 1. Let $(\bar{M}, \bar{g}, \bar{J})$ be an almost pseudo-Hermitian manifold and let $\theta \in \mathfrak{X}(\bar{M})$ be a complete strictly regular unit vector field $(\varepsilon=\bar{g}(\theta, \theta)= \pm 1)$. We consider that both $\bar{g}$ and $\bar{J}$ are invariant with respect to the uniparametric group $G$ defined by $\theta$. Then, the orbit space $(M, g, \phi, \xi, \eta)$ is an almost contact metric manifold, with

$$
g(X, Y)=\bar{g}\left(X^{H}, Y^{H}\right), \quad \phi X=\pi_{*}\left(\bar{J} X^{H}\right), \quad \xi=\pi_{*}(\bar{J} \theta),
$$

for any $X, Y \in T M$, where $X^{H}$ stands for the horizontal lift with respect to the mechanical connection, and $\eta$ is the dual form of $\xi$; that is, $\eta(\cdot)=\varepsilon g(\cdot, \xi)$.

Proof. As $\theta$ acts by preserving the metric and the complex structure tensor, we have that the tensors given in (5) are well defined. In addition, $\bar{J} \theta$, being orthogonal to $\theta$, is horizontal with respect to the mechanical connection and $g(\xi, \xi)=\bar{g}(\bar{J} \theta, \bar{J} \theta)=\bar{g}(\theta, \theta)=\varepsilon$. We have to check that

$$
\phi^{2}=-I d+\eta \otimes \xi, \quad g(\phi X, \phi Y)=g(X, Y)-\varepsilon \eta(X) \eta(Y) .
$$


If $X \in \xi^{\perp}=\{Y \in T M: g(Y, \xi)=0\}=\operatorname{ker} \eta$, then $\bar{g}\left(X^{H}, \bar{J} \theta\right)=0$, which means that $\bar{J}\left(X^{H}\right)$ is an horizontal vector. Then,

$$
\begin{aligned}
(\phi \circ \phi)(X) & =\phi\left(\pi_{*}\left(\bar{J} X^{H}\right)\right) \\
& =\pi_{*}\left(\left(\pi_{*}\left(\bar{J} X^{H}\right)\right)^{H}\right)=\pi_{*}\left(\bar{J}^{2} X^{H}\right)=-X .
\end{aligned}
$$

On the other hand, $\phi(\xi)=\pi_{*}\left(\bar{J}\left(\xi^{H}\right)\right)=\pi_{*}(\bar{J} \bar{\theta} \theta)=-\pi_{*}(\theta)=0$, so that both sides of

$$
(\phi \circ \phi)(\xi)=-\xi+\eta(\xi) \xi
$$

vanish. The first equation of (6) is satisfied.

With respect to the second equation, given $X \in T M$, we denote by $X^{\prime}$ the orthogonal part of $X$ with respect to $\xi$. Note that, since $\bar{g}\left(\bar{J}\left(X^{\prime H}\right), \theta\right)=-\bar{g}\left(X^{\prime H}, \bar{J} \theta\right)=g\left(X^{\prime}, \xi\right)=0$, the vector $\bar{J}\left(X^{\prime H}\right)$ is horizontal. Then

$$
\begin{aligned}
g(\phi X, \phi Y) & =g\left(\phi\left(X^{\prime}+\eta(X) \xi\right), \phi\left(Y^{\prime}+\eta(Y) \xi\right)\right)= \\
& =g\left(\phi\left(X^{\prime}\right), \phi\left(Y^{\prime}\right)\right)=\bar{g}\left(\bar{J}\left(X^{\prime H}\right), \bar{J}\left(Y^{\prime H}\right)\right) \\
& =\bar{g}\left(X^{\prime H}, Y^{\prime H}\right)=g\left(X^{\prime}, Y^{\prime}\right) \\
& =g(X, Y)-\varepsilon \eta(X) \eta(Y)
\end{aligned}
$$

and the proof is complete.

Remark 1. On top of the structure on the reduced manifold provided in the previous result, it is easy to check that the Levi-Civita connection on $M$ associated with $g$ is characterized by the condition

$$
\nabla_{X} Y=\pi_{*}\left(\bar{\nabla}_{X^{H}} Y^{H}\right), \quad X, Y \in \mathfrak{X}(M) .
$$

Associated with an almost contact metric manifold $(M, g, \phi, \xi, \eta)$, the canonical 2-form $\Phi$ is defined to be $\Phi(X, Y)=g(\phi(X), Y)$. Since

$$
\left(\widetilde{\nabla}_{X} \Phi\right)(Y, Z)=g\left(\left(\widetilde{\nabla}_{X} \phi\right) Y, Z\right), \quad X, Y, Z \in \mathfrak{X}(M),
$$

for any metric connection $\widetilde{\nabla}$, the manifold $M$ is cosymplectic if and only if $\nabla \Phi=0$. For non-cosymplectic manifolds, if we choose a point $p \in M, V=T_{p} M$, the 3-tensor $(\nabla \Phi)_{p}$ belongs to the space

$$
\mathcal{S}_{-}(V)=\left\{\alpha \in \mathcal{S}(V): \alpha_{X Y Z}=-\alpha_{X \phi Y \phi Z}+\eta(Y) \alpha_{X \xi Z}+\eta(Z) \alpha_{X Y \xi}\right\} .
$$

defined above. The classification of almost contact metric manifold in a category other than cosymplectic (Sasaki, trans-Sasaki, Kenmotsu, etc.; see [8]) is equivalent to $\nabla \Phi$ belonging to different combinations of the irreducible subspaces $\mathcal{C}_{1}, \ldots, \mathcal{C}_{12}$ in which $\mathcal{S}_{-}(V)$ decomposes. These subspaces can be organized in a less coarse classification

$$
\mathcal{S}_{-}(V)=\mathcal{S}_{-, 1}(V)+\mathcal{S}_{-, 0}(V)
$$

as

$$
\begin{aligned}
& \mathcal{S}_{-, 1}(V)=\mathcal{C}_{1} \oplus \mathcal{C}_{2} \oplus \mathcal{C}_{3} \oplus \mathcal{C}_{4} \oplus \mathcal{C}_{11} \\
& \mathcal{S}_{-, 0}(V)=\mathcal{C}_{5} \oplus \mathcal{C}_{6} \oplus \mathcal{C}_{7} \oplus \mathcal{C}_{8} \oplus \mathcal{C}_{9} \oplus \mathcal{C}_{10} \oplus \mathcal{C}_{12}
\end{aligned}
$$


and defined by the expressions

$$
\begin{aligned}
& \mathcal{S}_{-, 1}(V)=\left\{\alpha \in \mathcal{S}_{-}(V): \alpha_{X \xi Z}=0\right\}=\left\{\alpha \in \mathcal{S}_{-}(V): \alpha_{X Y Z}=-\alpha_{X \phi Y \phi Z}\right\}, \\
& \mathcal{S}_{-, 0}(V)=\left\{\alpha \in \mathcal{S}_{-}(V): \alpha_{X Y Z}=\eta(Y) \alpha_{X \zeta Z}+\eta(Z) \alpha_{X Y \xi}\right\} .
\end{aligned}
$$

Proposition 1. In the conditions of Theorem 1, if $(\bar{M}, \bar{g}, \bar{J})$ is a Kähler manifold, then the quotient space $(M, g, \phi, \xi, \eta)$ is an almost contact manifold such that $\nabla \Phi$ belongs to the class $\mathcal{S}_{-, 0}(V)=\mathcal{C}_{5} \oplus \mathcal{C}_{6} \oplus \mathcal{C}_{7} \oplus$ $\mathcal{C}_{8} \oplus \mathcal{C}_{9} \oplus \mathcal{C}_{10} \oplus \mathcal{C}_{12}$

Proof. For $X, Y, Z \in \mathfrak{X}(M)$ we have

$$
\begin{aligned}
\left(\nabla_{X} \phi\right) Y= & \nabla_{X}(\phi Y)-\phi\left(\nabla_{X} Y\right)=\pi_{*}\left(\bar{\nabla}_{X^{H}}(\phi Y)^{H}\right)-\pi_{*}\left(\bar{J}\left(\nabla_{X} Y\right)^{H}\right) \\
= & \pi_{*}\left(\bar{\nabla}_{X^{H}} J\left(Y^{\prime}\right)^{H}\right)-\pi_{*}\left(\bar{J}\left(\bar{\nabla}_{X^{H}} Y^{H}-\varepsilon \bar{g}\left(\bar{\nabla}_{X^{H}} Y^{H}, \theta\right) \theta\right)\right) \\
= & \pi_{*}\left(\bar{\nabla}_{X^{H}} J Y^{H}-\varepsilon \bar{\nabla}_{X^{H}} \bar{J}\left(\bar{g}\left(Y^{H}, \bar{J} \theta\right) \bar{J} \theta\right)\right. \\
& \left.\left.-\bar{J}\left(\bar{\nabla}_{X^{H}} Y^{H}\right)+\varepsilon \bar{g}\left(\bar{\nabla}_{X^{H}} Y^{H}, \theta\right) \bar{J} \theta\right)\right) .
\end{aligned}
$$

Since $\bar{\nabla} J=0$, the first and third terms of the last step above vanish and we get

$$
\begin{aligned}
\left(\nabla_{X} \phi\right) Y & \left.=\pi_{*}\left(\varepsilon X^{H}\left(\bar{g}\left(Y^{H}, \bar{J} \theta\right)\right) \theta\right)+\varepsilon \bar{g}\left(Y^{H}, \bar{J} \theta\right) \bar{\nabla}_{X^{H}} \theta+\varepsilon \bar{g}\left(\bar{\nabla}_{X^{H}} \bar{J} Y^{H}, \theta\right) \bar{J} \theta\right) \\
& =\eta(Y) \pi_{*}\left(\bar{\nabla}_{X^{H}} \theta\right)+\varepsilon \bar{g}\left(\bar{\nabla}_{X^{H}} Y^{H}, \theta\right) \xi
\end{aligned}
$$

so that

$$
g\left(\left(\nabla_{X} \phi\right) Y, Z\right)=\eta(Y) \bar{g}\left(\bar{\nabla}_{X^{H}} \theta, Z^{H}\right)+\eta(Z) \bar{g}\left(\bar{\nabla}_{X^{H}} Y^{H}, \theta\right),
$$

for any vector field $\mathfrak{X}(M)$. In particular,

$$
\begin{aligned}
g\left(\left(\nabla_{X} \phi\right) \xi, Z\right) & =\eta(\xi) \bar{g}\left(\bar{\nabla}_{X^{H}} \theta, Z^{H}\right)+\eta(Z) \bar{g}\left(\bar{\nabla}_{X^{H}} \xi^{H}, \theta\right) \\
& =\bar{g}\left(\bar{\nabla}_{X^{H}} \theta, Z^{H}\right)+\eta(Z) \bar{g}\left(\bar{\nabla}_{X^{H}} \bar{J} \theta, \theta\right)
\end{aligned}
$$

and

$$
\begin{aligned}
g\left(\left(\nabla_{X} \phi\right) Y, \xi\right) & =\eta(Y) \bar{g}\left(\bar{\nabla}_{X^{H}} \theta, \xi^{H}\right)+\eta(\xi) \bar{g}\left(\bar{\nabla}_{X^{H}} Y^{H}, \theta\right) \\
& =\eta(Y) \bar{g}\left(\bar{\nabla}_{X^{H}} \theta, \bar{J} \theta\right)+\bar{g}\left(\bar{\nabla}_{X^{H}} Y^{H}, \theta\right) \\
& =-\eta(Y) \bar{g}\left(\bar{\nabla}_{X^{H}} \bar{J} \theta, \theta\right)+\bar{g}\left(\bar{\nabla}_{X^{H}} Y^{H}, \theta\right) .
\end{aligned}
$$

Then

$$
\eta(Y) g\left(\left(\nabla_{X} \phi\right) \xi, Z\right)+\eta(Z) g\left(\left(\nabla_{X} \phi\right) Y, \xi\right)=\eta(Y) \bar{g}\left(\bar{\nabla}_{X^{H}} \theta, Z^{H}\right)+\eta(Z) \bar{g}\left(\bar{\nabla}_{X^{H}} Y^{H}, \theta\right) .
$$

Through a comparison with (7) we finally get

$$
g\left(\left(\nabla_{X} \phi\right) Y, Z\right)=\eta(Y) g\left(\left(\nabla_{X} \phi\right) \xi, Z\right)+\eta(Z) g\left(\left(\nabla_{X} \phi\right) Y, \xi\right) ;
$$

that is, $\nabla \Phi$ belongs to $\mathcal{S}_{-, 0}(V)$.

\section{Reduction of Homogeneous Structures}

We now assume that the pseudo-Hermitian manifold $(\bar{M}, \bar{g}, \bar{J})$ is equipped with a homogeneous structure tensor $\bar{S}$, which in addition is invariant under the action of the group $G$. 
Theorem 2. Let $(\bar{M}, \bar{g}, \bar{J})$ be an almost pseudo-Kähler manifold equipped with an almost pseudo-Kähler homogeneous structure $\bar{S}$ that is invariant under the group flow $G$ of a complete strictly regular unit vector field $\theta$. Suppose that

$$
\widetilde{\bar{\nabla}} \theta=\beta \otimes \theta
$$

where $\widetilde{\bar{\nabla}}=\bar{\nabla}-\bar{S}$ and $\beta$ is a 1-form on $M$. Then, the tensor field $S$ on the orbit space $M=\bar{M} / G$ defined by

$$
S_{X} Y=\pi_{*}\left(\bar{S}_{X^{H}} Y^{H}\right)
$$

is a homogeneous, almost contact metric structure on $(M, g, \phi, \xi, \eta)$ belonging to the class $\mathcal{S}_{+}(V) \oplus \mathcal{S}_{-, 0}(V)$. Furthermore, the components $S_{+} \in \mathcal{S}_{+}(V)$ and $S_{-, 0} \in \mathcal{S}_{-, 0}(V)$ of $S$ are

$$
\begin{aligned}
\left(S_{+}\right)_{X Y Z} & =S_{X \phi Y \phi Z} \\
\left(S_{-, 0}\right)_{X Y Z} & =\eta(Y) S_{X \xi Z}+\eta(Z) S_{X Y \xi},
\end{aligned}
$$

respectively.

Proof. Since the condition $\widetilde{\bar{\nabla}} \theta=\beta \otimes \theta$ is equivalent to $\widetilde{\bar{\nabla}} \omega=\beta \cdot \omega$, we are working in the conditions explained in Section 2.2 so that $S$ is a pseudo-Riemannian homogeneous structure. To show that $S$ is an almost contact metric homogeneous structure, we have to prove that $\widetilde{\nabla} \phi=0$, where $\widetilde{\nabla}=\nabla-S$. Let $X, Y \in \mathfrak{X}(M)$ be two vector fields,

$$
\omega\left(\widetilde{\bar{\nabla}}_{X^{H}} Y^{H}\right)=X^{H}\left(\omega\left(Y^{H}\right)\right)-\left(\widetilde{\bar{\nabla}}_{X^{H}} \omega\right)\left(Y^{H}\right)=-\beta\left(X^{H}\right) \omega\left(Y^{H}\right)=0
$$

so that $\widetilde{\bar{\nabla}}_{X^{H}} Y^{H}$ is horizontal. Then $\widetilde{\nabla}_{X^{H}} Y^{H}$ projects to $\widetilde{\nabla}_{X} Y$. Following the same steps in Proposition 1, we get

$$
g\left(\left(\widetilde{\nabla}_{X} \phi\right) Y, Z\right)=\eta(Y) \bar{g}\left(\widetilde{\bar{\nabla}}_{X^{H}} \theta, Z^{H}\right)+\eta(Z) \bar{g}\left(\widetilde{\bar{\nabla}}_{X^{H}} Y^{H}, \theta\right)
$$

which implies that $\widetilde{\nabla} \phi=0$, while again taking into consideration the fact that $\widetilde{\bar{\nabla}}_{X^{H}} Y^{H}$ is horizontal.

Now, we decompose $Y=Y^{\prime}+\eta(Y) \xi, Z=Z^{\prime}+\eta(Z) \xi$ and we get

$$
\begin{aligned}
S_{X Y Z} & =\bar{S}_{X^{H} Y^{H} Z^{H}} \\
& =\bar{S}_{X^{H}\left(Y^{\prime}\right)^{H}\left(Z^{\prime}\right)^{H}}+\eta(Y) \bar{S}_{X^{H} \bar{j} \theta\left(Z^{\prime}\right)^{H}}+\eta(Z) \bar{S}_{X^{H}\left(Y^{\prime}\right)^{H} \bar{j} \theta} \\
& =\bar{S}_{X^{H}\left(Y^{\prime}\right)^{H}\left(Z^{\prime}\right)^{H}}+\eta(Y) \bar{S}_{X^{H} \bar{j} \theta Z^{H}}+\eta(Z) \bar{S}_{X^{H} Y^{H} \bar{J} \theta} .
\end{aligned}
$$

Since $\bar{S}$ is a pseudo-Kähler homogeneous structure ,

$$
\begin{aligned}
S_{X Y Z} & =\bar{S}_{X^{H} \bar{J}\left(Y^{\prime}\right)^{H} \bar{J}\left(Z^{\prime}\right)^{H}}+\eta(Y) \bar{S}_{X^{H} \bar{\jmath} \theta Z^{H}}+\eta(Z) \bar{S}_{X^{H} Y^{H} \bar{\jmath} \theta} \\
& =S_{X \phi Y \phi Z}+\eta(Y) S_{X \zeta Z}+\eta(Z) S_{X Y \zeta^{\prime}}
\end{aligned}
$$

which implies that $S \in \mathcal{S}_{+}(V) \oplus \mathcal{S}_{-, 0}(V)$. Finally, it is a matter of direct checking that $S_{+}$and $S_{-, 0}$ in the statement satisfy $S_{+} \in \mathcal{S}_{+}(V)$ and $S_{-, 0} \in \mathcal{S}_{-, 0}(V)$.

Theorem 3. Let $(\bar{M}, \bar{g}, \bar{J})$ be a pseudo-Kähler manifold equipped with a pseudo-Kähler homogeneous structure $\bar{S}$ invariant under the flow group $G$ of a complete strictly regular unit vector field $\theta$. Assume that $\bar{S}$ belongs to the class $\mathcal{K}_{2}(\bar{V})+\mathcal{K}_{4}(\bar{V})$, parametrized by G-invariant vector fields $\chi_{2}$ and $\chi_{4}$. Then, the component 
$\left(S_{-, 0}\right) \in \mathcal{S}_{-, 0}(V)$ of the reduced homogeneous almost-contact metric structure $S$ of the almost contact metric manifold $(M=\bar{M} / G, g, \phi, \xi, \eta)$ belongs to $\mathcal{C}_{5}(V) \oplus \mathcal{C}_{6}(V) \oplus \mathcal{C}_{12}(V)$ with projections

$$
\begin{aligned}
\left(S_{-, 0}\right)_{(5)}(X, Y, Z) & =\varepsilon \omega(\chi)(\eta(Y) g(X, \phi Z)-\eta(Z) g(X, \phi Y)) \\
\left(S_{-, 0}\right)_{(6)}(X, Y, Z) & =\varepsilon \eta\left(\pi_{*} \chi\right)(\eta(Z) g(X, Y)-\eta(Y) g(X, Z)) \\
\left(S_{-, 0}\right)_{(12)}(X, Y, Z) & =\varepsilon \eta(X)\left(\eta(Y) g\left(Z, \pi_{*} \chi\right)-\eta(Z) g\left(Y, \pi_{*} \chi\right)\right)
\end{aligned}
$$

for $X, Y, Z \in V$, where $\chi=\chi_{2}+\chi_{4}$.

Proof. In the expression

$$
\left(S_{-, 0}\right)_{X Y Z}=\eta(Y) S_{X \xi Z}+\eta(Z) S_{X Y \xi}=\eta(Y) \bar{S}_{X^{H} \bar{j} \theta Z^{H}}+\eta(Z) \bar{S}_{X^{H} Y^{H} \bar{j} \theta}
$$

of the component $\mathcal{S}_{-, 0}(V)$ of $S$, we apply that $\bar{S} \in \mathcal{K}_{2}(\bar{V})+\mathcal{K}_{4}(\bar{V})$, defined by vector fields $\chi_{2}$ and $\chi_{4}$ (see Theorem A1), and we have

$$
\begin{aligned}
\left(S_{-, 0}\right)_{X Y Z}= & \eta(Y)\left(\bar{g}\left(X^{H}, \bar{J} \theta\right) \bar{g}\left(Z^{H}, \chi\right)-\bar{g}\left(X^{H}, Z^{H}\right) \bar{g}(\bar{J} \theta, \chi)\right. \\
& +\bar{g}\left(X^{H}, \bar{J}^{2} \theta\right) \bar{g}\left(\bar{J} Z^{H}, \chi\right)-\bar{g}\left(X^{H}, \bar{J} Z^{H}\right) \bar{g}\left(\bar{J}^{2} \theta, \chi\right) \\
& \left.-2 \bar{g}\left(\bar{J}^{2} \theta, Z^{H}\right) \bar{g}\left(\bar{J} X^{H}, \hat{\chi}\right)\right) \\
& +\eta(Z)\left(\bar{g}\left(X^{H}, Y^{H}\right) \bar{g}(\bar{J} \theta, \chi)-\bar{g}\left(X^{H}, \bar{J} \theta\right) \bar{g}\left(Y^{H}, \chi\right)\right. \\
& +\bar{g}\left(X^{H}, \bar{J} Y^{H}\right) \bar{g}\left(\bar{J}^{2} \theta, \chi\right)-\bar{g}\left(X^{H}, \bar{J}^{2} \theta\right) \bar{g}\left(\bar{J} Y^{H}, \chi\right) \\
& \left.-2 \bar{g}\left(\bar{J} Y^{H}, \bar{J} \theta\right) \bar{g}\left(\bar{J} X^{H}, \hat{\chi}\right)\right)
\end{aligned}
$$

where $\chi=\chi_{2}+\chi_{4}, \hat{\chi}=\chi_{2}-\chi_{4}$. As $\bar{g}\left(X^{H}, \theta\right)=0$ we get

$$
\begin{aligned}
\left(S_{-, 0}\right)_{X Y Z}= & \eta(Y)\left(\varepsilon \eta(X) g\left(Z, \pi_{*} \chi\right)-\varepsilon \eta\left(\pi_{*} \chi\right) g(X, Z)+\varepsilon g(X, \phi Z) \omega(\chi)\right) \\
& +\eta(Z)\left(\varepsilon \eta\left(\pi_{*} \chi\right) g(X, Y)-\varepsilon \eta(X) g\left(Y, \pi_{*} \chi\right)-\varepsilon g(X, \phi Y) \omega(\chi)\right) \\
= & \varepsilon \omega(\chi)(\eta(Y) g(X, \phi Z)-\eta(Z) g(x, \phi Y)) \\
& +\varepsilon \eta\left(\pi_{*} \chi\right)(\eta(Z) g(X, Y)-\eta(Y) g(X, Z)) \\
& +\varepsilon \eta(X)\left(\eta(Y) g\left(Z, \pi_{*} \chi\right)-\eta(Z) g\left(Y, \pi_{*} \phi\right)\right) .
\end{aligned}
$$

One can easily check from expression given in the Theorem A3 that first, second and third lines of the last equality belong to $\mathcal{C}_{5}(V), \mathcal{C}_{6}(V)$ and $\mathcal{C}_{12}(V)$ respectively.

Recall that the Ambrose-Singer condition $\widetilde{\nabla} \phi=0$ is equivalent to $\nabla \phi=[S, \phi]=\left[S_{-}, \phi\right]$. Hence

$$
\begin{aligned}
\left(\nabla_{X} \Phi\right)(Y, Z) & =g\left(\left(\nabla_{X} \phi\right) Y, Z\right)=g\left(\left(S_{-}\right)_{X}(\phi Y)-\phi\left(\left(S_{-}\right)_{X} Y\right), Z\right) \\
& =\left(S_{-}\right)_{X \phi Y Z}+\left(S_{-}\right)_{X Y} \phi Z
\end{aligned}
$$

If in addition $S_{-}$belongs to $\mathcal{S}_{-, 0}(V)$, then

$$
\begin{aligned}
\left(\nabla_{X} \Phi\right)(Y, Z) & =\eta(\phi Y)\left(S_{-}\right)_{X \zeta Z}+\eta Z\left(S_{-}\right)_{X \phi Y \xi}+\eta(Y)\left(S_{-}\right)_{X \zeta \phi Z}+\eta \phi Z\left(S_{-}\right)_{X Y \xi} \\
& =\eta(Z)\left(S_{-}\right)_{X \phi Y \xi}+\eta(Y)\left(S_{-}\right)_{X \xi \phi Z} .
\end{aligned}
$$


Proposition 2. Under the conditions of Theorem 3, the covariant derivative $\nabla \Phi$ of the fundamental form of the almost-contact metric manifold $(M, g, \phi, \xi, \eta)$ belongs to $\mathcal{C}_{5}(V) \oplus \mathcal{C}_{6}(V) \oplus \mathcal{C}_{12}(V)$ with components

$$
\begin{aligned}
\left(\left(\nabla_{X} \Phi\right)_{(5)}\right)(Y, Z) & =\varepsilon \eta\left(\pi_{*} \chi\right)(\eta(Z) g(X, \phi Y)-\eta(Y) g(X, \phi Z)) \\
\left(\left(\nabla_{X} \Phi\right)_{(6)}\right)(Y, Z) & =\varepsilon \omega(\chi)(\eta(Z) g(X, Y)-\eta(Y) g(X, Z)) \\
\left(\left(\nabla_{X} \Phi\right)_{(12)}\right)(Y, Z) & =\varepsilon \eta(X)\left(\eta(Y) g\left(\phi Z, \pi_{*} \chi\right)-\eta(Z) g\left(\phi Y, \pi_{*} \chi\right)\right)
\end{aligned}
$$

for $X, Y, Z \in \mathfrak{X}(M)$, where $\chi=\chi_{2}+\chi_{4}$.

Moreover, the manifold $(M, g, \phi, \xi, \eta)$ is cosymplectic if and only if $\chi=0$.

Proof. From Theorem 2, the part $S_{-}$of the reduced homogeneous structure $S_{\text {in }} \mathcal{S}_{-}(V)$ belongs to $\mathcal{S}_{-, 0}(V)$ and we can apply (8). By means of a straightforward computation, one can show that the components $\left(S_{-}\right)_{(5)},\left(S_{-}\right)_{(6)}$ and $\left(S_{-}\right)_{(12)}$ provide the expressions $\left(\nabla_{X} \Phi\right)_{(6)},\left(\nabla_{X} \Phi\right)_{(5)}$ and $\left(\nabla_{X} \Phi\right)_{(12)}$ in the statement, which belong to $\mathcal{C}_{6}(V), \mathcal{C}_{5}(V)$ and $\mathcal{C}_{12}(V)$ respectively. $\chi=0$.

Finally, the three components of $\nabla \Phi$ vanish if and only if $\pi_{*} \chi=0$ and $\omega(\chi)=0$, that is,

Corollary 1. Let $(\bar{M}, \bar{g}, \bar{J})$ be a pseudo-Kähler manifold equipped with a pseudo-Kähler homogeneous structure of the class $\mathcal{K}_{2}+\mathcal{K}_{4}$ defined by vector fields $\chi_{2}$ and $\chi_{4}$. Suppose that $\chi=\chi_{2}+\chi_{4}$ is a complete strictly regular vector field, and let $G$ be its flow group. Then the orbit manifold $(M=\bar{M} / G, g, \phi, \xi, \eta)$ is Sasakian.

Proof. The vector fields $\chi_{2}$ and $\chi_{4}$ satisfy $\widetilde{\bar{\nabla}} \chi_{2}=\widetilde{\bar{\nabla}} \chi_{4}=0$ (cf. [9]) so that $\chi=\chi_{2}+\chi_{4}$ satisfies the conditions of Theorem 2. From Proposition 2, since $\pi_{*} \chi=0$, we have that $\nabla \Phi$ belongs to the class $\mathcal{C}_{6}$, which is equivalent to being Sasakian ([8]).

Theorem 4. Let $(\bar{M}, \bar{g}, \bar{J})$ be a pseudo-Kähler manifold equipped with a pseudo-Kähler homogeneous structure $\bar{S}$ invariant under the flow group $G$ of a complete strictly regular unit vector field $\theta$. Assume that $\bar{S}$ belongs to the class $\mathcal{K}_{2}(\bar{V})+\mathcal{K}_{4}(\bar{V})$, parametrized by G-invariant vector fields $\chi_{2}$ and $\chi_{4}$. Then, the component $\left(S_{+}\right) \in \mathcal{S}_{+}(V)$ of the reduced homogeneous almost-contact metric structure $S$ of the almost contact metric manifold $(M=\bar{M} / G, g, \phi, \xi, \eta)$ belongs to $\mathcal{C S}_{2}(V) \oplus \mathcal{C S}_{4}(V) \oplus \mathcal{C S}_{6}(V)$, and its expression is

$$
\begin{aligned}
\left(S_{+}\right)(X, Y, Z)= & g(X, Y) g(Z, \rho)-\varepsilon \eta(X) \eta(Y) g(Z, \rho)-g(X, Z) g(Y, \rho) \\
& +\varepsilon \eta(X) \eta(Z) g(Y, \rho)+g(X, \phi Y) g(\phi Z, \rho)-g(X, \phi Z) g(\phi Y, \rho) \\
& -2 g(\phi Y, Z) g(\phi X, \hat{\rho})+2 \varepsilon \eta(X) \omega(\hat{\chi}) g(\phi Y, Z)
\end{aligned}
$$

for $X, Y, Z \in V$, where $\chi=\chi_{2}+\chi_{4}, \hat{\chi}=\chi_{2}-\chi_{4}$ and $\rho=\pi_{*} \chi-\eta\left(\pi_{*} \chi\right) \xi, \hat{\rho}=\pi_{*} \hat{\chi}-\eta\left(\pi_{*} \hat{\chi}\right) \xi$.

Proof. In the expression,

$$
\left(S_{+}\right)_{X Y Z}=S_{X \phi Y \phi Z}=\bar{S}_{X \bar{J} Y^{\prime H} \bar{J} Z^{\prime H}}=\bar{S}_{X Y^{\prime} Z^{\prime}}
$$

of the component $\mathcal{S}_{+}(V)$ of $S$, we apply the fact that $\bar{S} \in \mathcal{K}_{2}(\bar{V})+\mathcal{K}_{4}(\bar{V})$, defined by vector fields $\chi_{2}$ and $\chi_{4}$ (see Theorem A1), and we have

$$
\begin{aligned}
\left(S_{+}\right)_{X Y Z}= & \bar{g}\left(X^{H}, Y^{\prime H}\right) \bar{g}\left(Z^{\prime H}, \pi_{*} \chi\right)-\bar{g}\left(X^{H}, Z^{\prime H}\right) \bar{g}\left(Y^{\prime H}, \pi_{*} \chi\right) \\
& +\bar{g}\left(X^{H}, \bar{J} Y^{\prime H}\right) \bar{g}\left(\bar{J} Z^{\prime H}, \pi_{*} \chi\right)-\bar{g}\left(X^{H}, \bar{J} Z^{\prime H}\right) \bar{g}\left(\bar{J} Y^{\prime H}, \pi_{*} \chi\right) \\
& -2 \bar{g}\left(\bar{J} Y^{\prime H}, Z^{\prime H}\right) \bar{g}\left(\bar{J} X^{H}, \pi_{*} \hat{\chi}\right) \\
= & g\left(X, Y^{\prime}\right) g\left(Z^{\prime}, \pi_{*} \chi\right)-g\left(X, Z^{\prime}\right) g\left(Y^{\prime}, \pi_{*} \chi\right) \\
& +g(X, \phi Y) g\left(\phi Z, \pi_{*} \chi\right)-g(X, \phi Z) g\left(\phi Y, \pi_{*} \chi\right) \\
& -2 g\left(\phi Y, Z^{\prime}\right)\left(g\left(\phi X, \pi_{*} \hat{\chi}\right)-\varepsilon \eta(X) \omega(\hat{\chi})\right) .
\end{aligned}
$$


We use the fact that $g\left(Z^{\prime}, \pi_{*} \chi_{i}\right)=g\left(Z, \rho_{i}\right), g\left(\phi Y, \pi_{*} \chi_{i}\right)=g\left(\phi Y, \rho_{i}\right), g\left(\phi Y, Z^{\prime}\right)=g(\phi Y, Z)$ and we get

$$
\begin{aligned}
\left(S_{+}\right)_{X Y Z}= & g\left(X, Y^{\prime}\right) g(Z, \rho)-g\left(X, Z^{\prime}\right) g(Y, \rho) \\
& +g(X, \phi Y) g(\phi Z, \rho)-g(X, \phi Z) g(\phi Y, \rho) \\
& -2 g(\phi Y, Z) g(\phi X, \hat{\rho})+2 \varepsilon \eta(X) \omega(\hat{\chi}) g(\phi Y, Z) .
\end{aligned}
$$

Finally, taking into account that $g\left(X, Y^{\prime}\right)=g\left(X^{\prime}, Y\right), g\left(X, Z^{\prime}\right)=g\left(X^{\prime}, Z\right), X^{\prime}=X-\eta(X) \xi$, we get the given expression.

Corollary 2. Let $(\bar{M}, \bar{g}, \bar{J})$ be a pseudo-Kähler manifold equipped with a pseudo-Kähler homogeneous structure $\bar{S}$ invariant under the flow group $G$ of a complete strictly regular unit vector field $\theta$. Assume that $\bar{S}$ belongs to the class $\mathcal{K}_{2}(\bar{V})+\mathcal{K}_{4}(\bar{V})$. Then the reduced homogeneous almost-contact metric structure $S$ of the almost contact metric manifold $(M, g, \phi, \xi, \eta)$ is of linear type.

Proof. This result is a consequence of Theorems 3 and 4.

\section{Conclusions}

Since Ambrose-Singer [1] to the present, homogeneous structure tensors have been a main object in the study of the homogeneous manifolds with very interesting applications to other fields in differential geometry and theoretical physics, for example, those derived from linear structures. This paper is a contribution in those two lines of work:

- We showed a reduction procedure by one dimensional fibers between almost pseudo-Hermitian manifolds to almost contact metric manifolds of general type in the sense of Chinea-González (cf. [8]).

- We applied this fibration result to the case of homogeneous structures. In this context we got a reduction result between pseudo-Kähler homogeneous structures to almost contact metric homogeneous structures. Moreover, the reduced manifold lies in $\mathcal{C}_{5} \oplus \mathcal{C}_{6} \oplus \mathcal{C}_{7} \oplus \mathcal{C}_{8} \oplus \mathcal{C}_{9} \oplus \mathcal{C}_{10} \oplus \mathcal{C}_{12}$ of the Chinea-González classification (cf. [8]).

- We proved that the reduction procedure sends pseudo-Kähler homogeneous structures of linear type to almost contact metric homogeneous structures of linear type. Indeed, we showed the explicit expressions of the reduced homogeneous structure and gave a characterization of the reduced manifold being cosymplectic and Sasakian.

- The study of homogeneous structures of linear type is connected with models of singular plane waves in general relativity (see [15] for the real case and [16] for the pseudo-Kähler setting explored in this work). The models associated with the particular instances that arose from our results, along with some other examples, will be the topic of future research.

Furthermore, we can conclude once more that homogeneous structures have an important and restrictive influence on the geometric structures of the manifold (for example, being xosymplectic or Sasakian). In our opinion, that makes homogeneous structures a fruitful main tool in the study of homogeneous manifolds.

Author Contributions: All authors contributed equally and significantly to writing this article. All authors have read and agreed to the published version of the manuscript.

Funding: Both authors have been partially supported by MICINN (Spain) under grant number PGC2018-098321-B-I00. M.C.L. has been partially supported by Junta de Castilla y León (Spain) under grant number SA090G19. J.L.C.J. acknowledge financial support from "Severo Ochoa Programme for Centres of Excellence in R\&D" (SEV-2015-0554) and from the Spanish National Research Council, through the "Ayuda extraordinaria a Centros de Excelencia Severo Ochoa" (20205- CEX001).

Conflicts of Interest: The authors declare no conflict of interest. 


\section{Appendix A. Expressions of Homogeneous Structures}

We now give the explicit expressions of the classes given in (2)-(4) and their corresponding classification theorems.

Theorem A1 ([4]). If $m \geq 6$, the space $\mathcal{K}(V)$ is decomposed into mutually orthogonal and irreducible $\mathrm{U}(p, q)$-submodules as

$$
\mathcal{K}(V)=\mathcal{K}_{1}(V) \oplus \mathcal{K}_{2}(V) \oplus \mathcal{K}_{3}(V) \oplus \mathcal{K}_{4}(V),
$$

where

$$
\begin{gathered}
\mathcal{K}_{1}(V)=\left\{S \in \mathcal{K}(V): S_{X Y Z}=\frac{1}{2}\left(S_{Y Z X}+S_{Z X Y}+S_{J Y J Z X}+S_{J Z X J Y}\right),\right. \\
\left.c_{12}(S)=0\right\}, \\
\mathcal{K}_{2}(V)=\left\{S \in \mathcal{K}(V): S_{X Y Z}=\langle X, Y\rangle \chi_{2}(Z)-\langle X, Z\rangle \chi_{2}(Y)+\langle X, J Y\rangle \chi_{2}(J Z)\right. \\
\left.-\langle X, J Z\rangle \chi_{2}(J Y)-2\langle J Y, Z\rangle \chi_{2}(J X), \chi_{2} \in V^{*}\right\}, \\
\mathcal{K}_{3}(V)=\left\{S \in \mathcal{K}(V): S_{X Y Z}=-\frac{1}{2}\left(S_{Y Z X}+S_{Z X Y}+S_{J Y J Z X}+S_{J Z X J Y}\right),\right. \\
\left.\mathrm{c}_{12}(S)=0\right\}, \\
\mathcal{K}_{4}(V)=\left\{S \in \mathcal{K}(V): S_{X Y Z}=\langle X, Y\rangle \chi_{4}(Z)-\langle X, Z\rangle \chi_{4}(Y)+\langle X, J Y\rangle \chi_{4}(J Z)\right. \\
\left.-\langle X, J Z\rangle \chi_{4}(J Y)-2\langle J Y, Z\rangle \chi_{4}(J X), \chi_{4} \in V^{*}\right\}
\end{gathered}
$$

and

$$
\mathrm{c}_{12}(S)(Z)=\sum_{i=1}^{m}\left(S_{e_{i} e_{i} Z}\right)
$$

for an orthonormal basis $\left\{e_{1}, \ldots, e_{m}\right\}$.

Theorem A2 ([5]). If $m \geq 7$, the space $\mathcal{S}_{+}(V)$ decomposes into irreducible and mutually orthogonal $\mathrm{U}(p, q) \times$ $\{1\}$-submodules as

$$
\mathcal{S}_{+}(V)=\mathcal{C S}_{1}(V) \oplus \mathcal{C} \mathcal{S}_{2}(V) \oplus \mathcal{C S}_{3}(V) \oplus \mathcal{C S}_{4}(V) \oplus \mathcal{C S}_{5}(V) \oplus \mathcal{C S}_{6}(V)
$$

where

$$
\begin{aligned}
& \mathcal{C S}_{1}(V)=\left\{S \in \mathcal{S}_{+}(V): S_{X Y Z}=\frac{1}{2}\left(S_{Y Z X}+S_{Z X Y}+S_{J Y J Z X}+S_{J Z X J Y}\right),\right. \\
& \left.\mathrm{c}_{12}(S)=0\right\}, \\
& \mathcal{C S}_{2}(V)=\left\{S \in \mathcal{S}_{+}(V): S_{X Y Z}=\langle X, Y\rangle \psi_{2}(Z)-\varepsilon \eta(X) \eta(Y) \psi_{2}(Z)-\langle X, Z\rangle \psi_{2}(Y)\right. \\
& +\varepsilon \eta(X) \eta(Z) \psi_{2}(Y)+\langle X, \phi Y\rangle \psi_{2}(\phi Z)-\langle X, \phi Z\rangle \psi_{2}(\phi Y) \\
& \left.-2\langle\phi Y, Z\rangle \psi_{2}(\phi X), \psi_{2} \in \hat{V}^{*}\right\} \text {, } \\
& \mathcal{C S}_{3}(V)=\left\{S \in \mathcal{S}_{+}(V): S_{X Y Z}=-\frac{1}{2}\left(S_{Y Z X}+S_{Z X Y}+S_{J Y J Z X}+S_{J Z X Y Y}\right),\right. \\
& \left.\mathrm{c}_{12}(S)=0\right\}, \\
& \mathcal{C S}_{4}(V)=\left\{S \in \mathcal{S}_{+}(V): S_{X Y Z}=\langle X, Y\rangle \psi_{4}(Z)-\varepsilon \eta(X) \eta(Y) \psi_{4}(Z)-\langle X, Z\rangle \psi_{4}(Y)\right. \\
& +\varepsilon \eta(X) \eta(Z) \psi_{4}(Y)+\langle X, \phi Y\rangle \psi_{4}(\phi Z)-\langle X, \phi Z\rangle \psi_{4}(\phi Y) \\
& \left.+2\langle\phi Y, Z\rangle \psi_{4}(\phi X), \psi_{4} \in \hat{V}^{*}\right\}, \\
& \mathcal{C S}_{5}(V)=\left\{S \in \mathcal{S}_{+}(V): S_{X Y Z}=\alpha \eta(X) g(Y, \phi Z), \alpha \in \mathbb{R}\right\}, \\
& \mathcal{C S}_{6}(V)=\left\{S \in \mathcal{S}_{+}(V): c_{2 \phi 3}(\xi)=0\right\},
\end{aligned}
$$


and

$$
\mathrm{c}_{12}(S)(Z)=\sum_{i=1}^{m-1} S_{e_{i} e_{i} Z}, \quad c_{2 \phi 3}(S)(Z)=\sum_{i=1}^{m-1} S_{Z e_{i} \phi e_{i}}
$$

for $\hat{V}$ the orthogonal complement to $\xi$, an orthonormal basis $\left\{e_{1}, \ldots, e_{m-1}\right\}$ of $\hat{V}$ and $\varepsilon=g(\xi, \xi)$.

Theorem A3 ([8]). If $m \geq 7$, the space $\mathcal{S}_{-}(V)$ decomposes into irreducible and mutually orthogonal $\mathrm{U}(p, q) \times$ $\{1\}$-submodules as

$$
\mathcal{S}_{-}(V)=\mathcal{C}_{1}(V) \oplus \cdots \oplus \mathcal{C}_{12}(V)
$$

where

$$
\begin{aligned}
& \mathcal{C}_{1}(V)=\left\{S \in \mathcal{S}_{-}(V): S_{X X Y}=S_{X Y \xi}=0\right\}, \\
& \mathcal{C}_{2}(V)=\left\{S \in \mathcal{S}_{-}(V): \mathfrak{S}_{X, Y, Z} S_{X Y Z}=0, S_{X Y \xi}=0\right\}, \\
& \mathcal{C}_{3}(V)=\left\{S \in \mathcal{S}_{-}(V): S_{X Y Z}-S_{\phi X \phi Y Z}=0, \mathrm{c}_{12}(S)=0\right\}, \\
& \mathcal{C}_{4}(V)=\left\{S \in \mathcal{S}_{-}(V): S_{X Y Z}=\langle X, Y\rangle \mu_{4}(Z)-\varepsilon \eta(X) \eta(Y) \mu_{4}(Z)-\langle X, Z\rangle \mu_{4}(Y)\right. \\
& \left.+\varepsilon \eta(X) \eta(Z) \mu_{4}(Y)-\langle X, \phi Y\rangle \mu_{4}(\phi Z)+\langle X, \phi Z\rangle \mu_{4}(\phi Y), \mu_{4} \in \hat{V}^{*}\right\}, \\
& \mathcal{C}_{5}(V)=\left\{S \in \mathcal{S}_{-}(V): S_{X Y Z}=\beta \varepsilon(\eta(Y) g(X, \phi Z)-\eta(Z) g(X, \phi Y)), \beta \in \mathbb{R}\right\}, \\
& \mathcal{C}_{6}(V)=\left\{S \in \mathcal{S}_{-}(V): S_{X Y Z}=\gamma \mathcal{E}(\eta(Y) g(X, Z)-\eta(Z) g(X, Y)), \gamma \in \mathbb{R}\right\}, \\
& \mathcal{C}_{7}(V)=\left\{S \in \mathcal{S}_{-}(V): S_{X Y Z}=\eta(Z) S_{Y X \xi}-\eta(Y) S_{\phi X \phi Z \xi}, c_{12}(S)(\xi)=0\right\}, \\
& \mathcal{C}_{8}(V)=\left\{S \in \mathcal{S}_{-}(V): S_{X Y Z}=-\eta(Z) S_{Y X \xi}-\eta(Y) S_{\phi X \phi Z \xi}, \mathrm{c}_{1 \phi 2}(S)(\xi)=0\right\}, \\
& \mathcal{C}_{9}(V)=\left\{S \in \mathcal{S}_{-}(V): S_{X Y Z}=\eta(Z) S_{Y X \xi}+\eta(Y) S_{\phi X \phi Z \xi}\right\}, \\
& \mathcal{C}_{10}(V)=\left\{S \in \mathcal{S}_{-}(V): S_{X Y Z}=-\eta(Z) S_{Y X \xi}+\eta(Y) S_{\phi X \phi Z \xi}\right\}, \\
& \mathcal{C}_{11}(V)=\left\{S \in \mathcal{S}_{-}(V): S_{X Y Z}=-\eta(X) S_{\tilde{\zeta} \phi Y \phi Z}\right\}, \\
& \mathcal{C}_{12}(V)=\left\{S \in \mathcal{S}_{-}(V): S_{X Y Z}=\varepsilon \eta(X)\left(\eta(Y) \mu_{12}(Z)-\eta(Z) \mu_{12}(Y)\right), \mu_{12} \in \hat{V}^{*}\right\},
\end{aligned}
$$

and

$$
\mathrm{c}_{12}(S)(Z)=\sum_{i=1}^{m-1} S_{e_{i} e_{i} Z}, \quad c_{1 \phi 2}(S)(Z)=\sum_{i=1}^{m-1} S_{e_{i} \phi e_{i} Z}
$$

for $\hat{V}$ the orthogonal complement to $\xi$, an orthonormal basis $\left\{e_{1}, \ldots, e_{m-1}\right\}$ of $\hat{V}$ and $\varepsilon=g(\xi, \xi)$.

\section{References}

1. Ambrose, W.; Singer, I.M. On homogeneous Riemannian manifolds. Duke Math. J. 1958, 25, 647-669. [CrossRef]

2. Kiričenko, V.F. On homogeneous Riemannian spaces with an invariant structure tensor. Sov. Math. Dokl. 1980, 21, 734-737.

3. Tricerri, F.; Tricerri, G.; Vanhecke, L. Homogeneous Structures on Riemannian Manifolds, 1st ed.; Lon. Math. Soc. Lecture Notes Series 83; Cambridge University Press: London, UK, 1983.

4. Abbena, E.; Garbiero, S. Almost hermitian homogeneous structures. Proc. Edinb. Math. Soc. 1988, 31, 375-395. [CrossRef]

5. Calvaruso, G.; Castrillón López, M. Pseudo-Riemannian Homogeneous Structures, 1st ed.; Developments in Mathematics 59; Springer: New York, NY, USA, 2019.

6. Castrillón López, M.; Luján, I. Homogeneous structures of linear type on $\epsilon$-Kähler and $\epsilon$ - quaternion Kähler manifolds Rev. Mat. Iberoam. 2017, 33, 139-168. [CrossRef]

7. Castrillón López, M.; Luján, I. Reduction of homogeneous Riemannian structures. Proc. Edinh. Math. Soc. 2015, 58, 81-106. [CrossRef] 
8. Chinea, D.; González, C. A Classification of Almost Contact Metric Manifolds. Annali di Matematica Pura ed Applicata 1990, 156, 15-36. [CrossRef]

9. Batat, W.; Gadea, P.; Oubiña, J.A. Homogeneous pseudo-Riemannian structures of linear type. J. Geom. Phys. 2011, 60, 745-764. [CrossRef]

10. Carmona Jiménez, J.L.; Castrillón López, M. The Ambrose-Singer theorem for general homogeneous spaces with applications to symplectic geometry. arXiv 2020, unpublished. Available online: https://arxiv.org/abs/ 2001.06254 (accessed on 17 January 2020).

11. Luján. I. Reductive locally homogeneous pseudo-Riemannian manifolds and Ambrose-Singer connections. Diff. Geom. Appl. 2015, 41, 65-90. [CrossRef]

12. Blair, D.E. Riemannian Geometry of Contact and Symplectic Manifolds, 1st ed.; Progress in Mathematics 203; Birkhäuser: Basel, Switzerland, 2002.

13. Marsden, J.E.; Ostrowski, J. Symmetries in motion: Geometric foundations of motion control. Nonlinear Sci. Today 1996, 1-21.

14. Palais, R. A Global Formulation of the Lie Theory of Transformation Groups; Mem. Amer. Math. Soc. 22; American Mathematical Society: Providence, RI, USA, 1957.

15. Meessen, P. Homogeneous Lorentzian spaces admitting a homogeneous structure of type $\mathcal{T}_{1}+\mathcal{T}_{2}$. J. Geom. Phys. 2006, 56, 754-761. [CrossRef]

16. Castrillón López, M.; Luján, I. Strongly degenerate homogeneous pseudo-Kähler structures of linear type and complex plane waves. J. Geom. Phys. 2013, 73, 1-19. [CrossRef]

(C) 2020 by the authors. Licensee MDPI, Basel, Switzerland. This article is an open access article distributed under the terms and conditions of the Creative Commons Attribution (CC BY) license (http:/ / creativecommons.org/licenses/by/4.0/). 